\title{
AVALIAÇÃO PREVENTIVA DA DISFUNÇÃO TEMPOROMANDIBULAR
}

Letícia Stadela da Silva ${ }^{1}$, Lisyara Helena Tori Barra ${ }^{1}$, Célia Aparecida Stellutti Pachioni ${ }^{1}$, Dalva Minonroze Albuquerque Ferreira ${ }^{1}$, João Domingos Augusto dos Santos Pereira ${ }^{2}$

${ }^{1}$ Departamento de Fisioterapia e ${ }^{2}$ Departamento de Física, Química e Biologia da FCT/UNESP, Presidente Prudente, SP. leticiastadela@hotmail.com

\section{RESUMO}

O objetivo deste trabalho foi detectar precocemente através de questionários específicos, sinais e sintomas de DTM. Foram avaliados 50 estudantes, de ambos os gêneros e faixa etária entre 18 e 30 anos por meio dos questionários: Critérios de Diagnóstico para Pesquisa das Disfunções Temporomandibulares (RDC/TMD) eixos I e II para diagnóstico e para avaliar a severidade da disfunção utilizou-se o Índice clínico de Fonseca. A análise estatística descritiva dos dados mostrou que dos 50 sujeitos, 19 foram diagnosticados com DTM, ou seja, 38\%. Foi verificada prevalência de DTM no gênero feminino $(40,5 \%)$ e no gênero masculino $(30,7 \%)$. Dentre os sujeitos com diagnóstico de DTM, obtiveram as mesmas porcentagens tanto para DTM artrogênica como miogênica (36,8\%) e 26,4\% DTM mista. Podemos concluir que a DTM não foi prevalente nos universitários sendo na maioria classificada de grau leve, mostrando-se necessária uma possível intervenção para que a mesma não se agrave.

Palavras-chave: disfunção, temporomandibular, questionário.

\section{PREVENTIVE EVALUATION OF TEMPOROMANDIBULAR DISORDERS}

\begin{abstract}
The aim of this study was early detection of TMD`s signs and symptoms through specific questionnaires. Fifty students were evaluated of both sexes and aged between 18 and 30 years through the questionnaires: for the diagnosis it was used the Diagnostic Criteria for Research of Temporomandibular Disorders (RDC I TMD) axis I and II and; to assess the severity of the disorder the chosen was the Fonseca's Clinical Index. Descriptive statistical analysis showed that of 50 subjects, $19(38 \%)$ were diagnosed with TMD. The prevalence of TMD was found in females (40.5\%) and in males (30.7\%). Among the subjects with diagnosis of TMD, arthrogenous as myogenic obtained the same percentages (36.8\%) for both TMD and a percentage of 26.4\%to mixed TMD. We conclude that TMD was not prevalent in college, in most classified with mild level, showing a possible necessary intervention to prevent it becoming worse.
\end{abstract}

Keywords: dysfunction, temporomandibular, questionnaire. 


\section{INTRODUÇÃO}

A Articulação Temporomandibular (ATM) é um elemento do sistema estomatognático formado por várias estruturas internas e externas, capaz de realizar movimentos complexos. A mastigação, a deglutição, a fonação e a postura, dependem muito da função, saúde e estabilidade desta para funcionarem de forma adequada ${ }^{1}$.

A disfunção Temporomandibular (DTM) é definida como um conjunto de condições médicas, dentárias ou faciais associadas com anormalidades do sistema estomatognático, que desencadeiam disfunções na articulação temporomandibular e tecidos adjacentes, incluindo os músculos faciais e cervicais².

Três grupos principais de fatores etiológicos estão envolvidos no aparecimento da DTM: fatores anatômicos, fatores neuromusculares e fatores psicogênicos. Se dois ou três destes grupos de fatores estiverem presentes, o risco de desenvolver a dor e a disfunção aumenta ${ }^{3}$

Os principais sinais e sintomas associados à DTM são: dor intra-articular, espasmo muscular, dor intra-articular combinada com espasmos musculares, dor reflexa, dor na abertura e fechamento da mandíbula, dor irradiada na área temporal, masseteriana ou infraorbital; crepitação, dor ou zumbido no ouvido; dor irradiada no pescoço; dor de cabeça crônica; sensação de tamponamento do ouvido; entre outros ${ }^{4}$.

Em virtude da complexidade etiológica e da variedade dos sinais e sintomas que podem, genericamente, também representar outras patologias, o reconhecimento e a diferenciação das DTMs podem apresentar-se de forma não muito clara ao profissional. Sendo assim, se torna necessária avaliação precoce por meio de questionários que possibilite a caracterização dos portadores de DTM.

\section{OBJETIVO}

O objetivo do trabalho é: detectar precocemente através de questionários específicos, sinais e sintomas de DTM.

\section{METODOLOGIA}

\section{Sujeitos}

Foram avaliados 50 estudantes da Universidade Estadual Paulista "Júlio de Mesquita Filho" campus de Presidente Prudente, de ambos os sexos e com faixa etária entre 18 e 35 anos. Este trabalho foi aprovado pelo Comitê de Ética em Pesquisa da Faculdade de Ciências e Tecnologia da UNESP - Campus de Presidente Prudente sob o protocolo 51/2009.

\section{Materiais e Métodos}

Foi aplicado aos sujeitos da pesquisa, o questionário: Critérios de Diagnóstico para Pesquisa das Disfunções Temporomandibulares (RDC/TMD) o qual contém questões relacionadas aos dados pessoais, saúde oral, hábitos deletérios, ruídos articulares, sintomas auditivos e psicológicos. O RDC/TMD envolve uma abordagem biaxial como uma tentativa inicial de permitir uma mensuração confiável de achados físicos, e avaliação confiável do status psicossocial que se envolve um perfil de disfunção dolorosa crônica, depressão, ansiedade e preocupação com outros sintomas físicos, além de incluir uma avaliação de limitações na habilidade normal durante função mandibular ${ }^{5}$.

Os sujeitos com diagnóstico de DTM também foram submetidos ao Índice Clínico de Fonseca ${ }^{6}$, constituído de dez questões. Este questionário é uma ferramenta importante para determinar o grau de severidade da DTM.

\section{RESULTADOS}

Neste estudo foram avaliados 50 sujeitos, 13 do gênero masculino e 37 do gênero feminino, sendo que 19 sujeitos foram diagnosticados com 
DTM, ou seja, 38\%. Foi verificada maior prevalência de DTM no sexo feminino (40,5\%), sendo 30,7\% no gênero masculino. Verificou-se que 57,8\% apresentam DTM leve; 26,3\% DTM moderada e 15,7\% severa. A classificação em DTM severa, foi predominante apenas no sexo feminino (20\%).

Dentre os sujeitos com diagnóstico de DTM, verificaram-se mesmos valores tanto para DTM artrogênica como miogênica (36,8\%) e 26,4\% DTM mista.

A maior parte da amostra não apresentou nenhum tipo de ruídos articulares, tais como: crepitação fina, crepitação grosseira e estalido, porém dentre os sujeitos que apresentaram, constatamos que o mais prevalente em ambas as ATMs foi a crepitação fina durante os movimentos de: abertura, fechamento, excursões laterais e protrusão da mandíbula.

Com relação à palpação, nos músculos extraorais, houve maior queixa de dor na palpação do masseter médio (40\%), masseter inferior (34\%) e na região mandibular posterior, a qual envolve os músculos estilo-hióideo e digástrico (36\%). Na palpação articular, a dor foi mais freqüente na região do pólo lateral da ATM aparecendo somente em 8\% dos sujeitos. Nos músculos intraorais a incidência de dor foi igual tanto para a área do músculo pterigóideo lateral, quanto para o tendão do músculo temporal, onde $54 \%$ dos sujeitos relataram dor.

No eixo II pôde ser observado que 68,4\% dos indivíduos com DTM relataram ter sentido dor na face, nos lados da cabeça, na frente do ouvido ou no ouvido no último mês com relação à aplicação do questionário RDC/TMD; sendo que destes apenas 15,7 \% procuraram ajuda com algum profissional da saúde; 52,6\% relataram problemas com dores de cabeça e enxaquecas nos últimos seis meses.

Em relação às atividades que foram limitadas devido à dor na face ou alguma alteração na mandíbula, 52,6\% relataram maiores prejuízos ao bocejar e ao comer alimentos duros; $36,8 \%$ ao mastigar; 5,2\% ao limpar os dentes ou face e 5,4\% não relataram nenhum tipo de prejuízo. Dentre estas atividades houve diferença significativa entre o gênero masculino e feminino.

Já em relação aos principais sinais observados pelos indivíduos, 78,9\% relataram ouvir estalos ao mastigar ou ao abrir ou fechar a boca; $68,4 \%$ ouvem um rangido ao mastigar ou abrir a boca e 57,9\% sente a mandíbula fadigada ou dolorida ao acordar. $\mathrm{Na}$ questão sobre bruxismo e briquismo, 47,3\% relataram ter tais hábitos. Foi relatado também que 42,1\% ouvem zumbidos e sentem que os dentes não se articulam de maneira correta. O estalo foi o sinal mais relatado em ambos os gêneros, e mais freqüente no gênero masculino (100 \%).

Por fim, os aspectos psicológicos mais encontrados foram: $68,4 \%$ disseram sentir dores na parte inferior da coluna e também a mesma porcentagem relatou se preocupar muito; 57,9\% relataram ter músculos doloridos e 52,6\% têm uma sensação de falta de energia e lentidão. Foi observado que o gênero masculino é mais influenciado por fatores psicológicos (Figura 1).

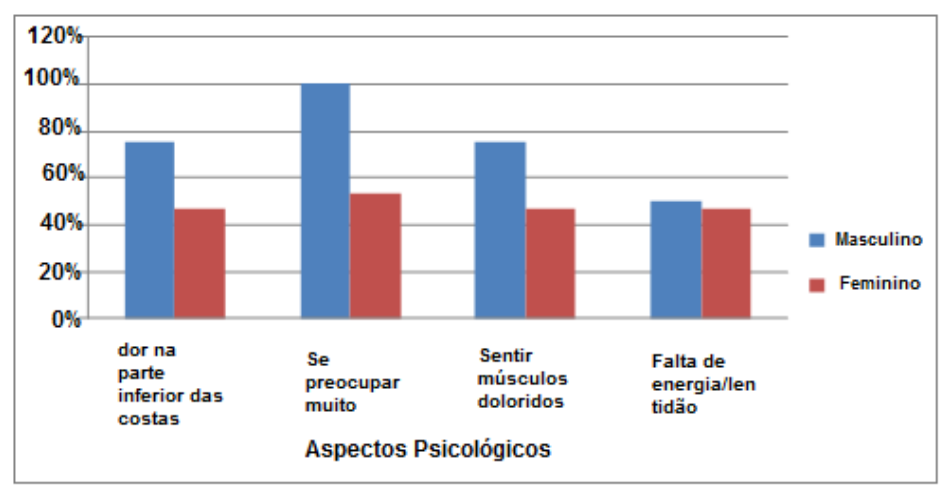

Figura 1. Distribuição em porcentagem dos principais aspectos psicológicos relatados pelos universitários. 


\section{DISCUSSÃO}

O presente estudo foi realizado com 50 estudantes de ambos os gêneros e com faixa etária entre 18 e 35 anos. Corroborando com outros estudos ${ }^{7,8}$ houve maior prevalência do gênero feminino, que pode ser atribuída devido a correlações entre a DTM e influências hormonais visto que suas manifestações são mais freqüentes no sexo feminino. Além disso, outros autores relatam que as mulheres são mais cuidadosas e com maior atenção à sua saúde, respondendo melhor às questões do questionário RDC/TMD por possuir um melhor autoconhecimento do que os homens ${ }^{9}$.

Em relação ao grau de severidade, a DTM leve foi a mais freqüente nos universitários consoante aos estudos de Vieira et al. ${ }^{10}$. Fato este que pode ser explicado devido à média dos participantes da pesquisa ser jovem, podendo assim apresentar algum grau de disfunção, porém ainda não severo, ressaltando a importância da avaliação precoce.

Verificamos que a média de abertura máxima foi de 47, $660 \mathrm{~mm}$, e a média para excursão lateral da mandíbula direita e esquerda foi $>9 \mathrm{~mm}$, sendo considerados valores dentro da normalidade $^{11}$. Tais valores se contrapõem a maioria dos achados descritos para as disfunções articulares $^{12,13}$.

Com relação aos ruídos articulares durante os movimentos mandibulares, foi constatado no exame físico presença de crepitação fina na maior parte dos sujeitos, porém quando os mesmos eram questionados sobre a percepção desses ruídos, foi referida maior presença de estalido. A maior percepção de estalido pelos sujeitos já foi descrita na literatura, que também descreve que os estalidos são um dos sintomas mais freqüentes em pacientes com DTM, e ocorrem devido ao posicionamento incorreto da cartilagem ${ }^{14}$.
Durante a palpação o músculo com maior queixa de dor foi o masseter, seguido da região mandibular posterior, a qual envolve os músculos estilo-hióideo e digástrico. Tal achado não está de acordo com a literatura, que mostra resultados referentes ao músculo pterigóideo lateral e ao músculo temporal como os mais afetados pela dor $^{15}$.

Quando questionados sobre as queixas de dor, as mais citadas foram de dores na região da face, no ouvido, cefaléias, sintomas estes que também foram verificados nos estudos de Okeson $^{16}$. As dificuldades encontradas ao realizar movimentos mandibulares relatadas pelos universitários, como mastigar, bocejar foram constatados também nos estudos de Felício et al. ${ }^{17}$. Comer alimentos duros, também foi citado como uma atividade limitada pela dor, um dos motivos possíveis para tal dificuldade é que a freqüência deste hábito promove desgaste fisiológico dos dentes contribuindo para piora da oclusão, gerando excesso mastigatório unilateral, interferindo na posição do côndilo da mandíbula, causando alterações, como relatado por $\mathrm{Melo}^{18}$, 1998.

Em relação à percepção dos principais sintomas referidos pelos participantes da pesquisa, a presença de estalos ao abrir e fechar a boca ou mastigar foi bastante freqüente em ambos os gêneros, sendo também constada em diversos trabalhos ${ }^{19,20}$.

Outro achado muito relevante foi com relação aos hábitos parafuncionais, como o bruxismo e o briquismo, que podem ser relacionados com o fato de grande parte dos sujeitos relatarem sentir a mandíbula fadigada ou dolorida pela manhã, bem como por se tratar de uma população jovem o fator emocional também pode estar implícito nesta questão. Tais resultados podem ser confirmados também em outros achados ${ }^{8,10,17}$. 
Bove et al. ${ }^{21}$, relataram a presença de dores em várias regiões do corpo e também sintomas emocionais de ansiedade nos indivíduos portadores de DTM e que se pode verificar exagerada tensão, perfeccionismo, ambição que podem ser expressas, entre outras formas, através de parafunções ou hábitos orais.Tal fato pode explicar os resultados deste estudo, onde os indivíduos relataram sentir dores na parte inferior da coluna e nos músculos além de se preocupar muito com as coisas e ter uma sensação de falta de energia e lentidão.

\section{CONCLUSÃO}

Podemos concluir com o presente estudo, que a utilização de questionários específicos é eficaz para detectar a presença de sinais e sintomas da Disfunção Temporomandibular, tornando possível o diagnóstico precoce e portando prevenindo futuras degenerações. Também foi possível observar que a DTM não foi prevalente nos universitários e que dentre os sujeitos diagnosticados, prevaleceu grau leve de DTM, mostrando-se necessária uma possível intervenção para que a mesma não se agrave. Além disso, evidenciou-se que o aspecto emocional pode ser considerado um fator desencadeante da disfunção, ainda mais no presente estudo, onde o ambiente universitário oferece situações estressantes.

\section{REFERÊNCIAS}

1. Quinto CA. Classificação e tratamento das disfunções temporomandibulares: qual o papel do fonoaudiólogo no tratamento dessas disfunções? Revista CEFAC 2000;2(2):15-22.

2. Piozzi R, Lopes FC. Desordens temporomandibulares: aspectos clínicos e guia para a odontologia e fisioterapia. Jornal Brasileiro de Oclusão, ATM e Dor Orofacial 2002;2(5):43-7.
3. Zarb GA, Carlsson GE, Sessle BJ, Mohl OD. Disfunções da articulação temporomandibular e dos músculos da mastigação. São Paulo; 2000.

4. Meira GSP. DTM $\times$ problemas otológicos [Internet]. 2002. [Acesso em 2008 out 10]. Disponível em: http://www.aonp.org.br/fso/revista7/rev712a.htm

5. Dworkin SF, Le Resche L. Critério de diagnóstico em pesquisa para desordens temporomandibulares: revisão, exames e especificações, crítica, 1992. In: Fricton, J.R. Dor orofacial e desordens temporomandibulares, 2003. (faltam as páginas)

6. Fonseca DM, Bonfante G, Valle AL, Freitas SFT. Diagnóstico pela anamnese da disfunção craniomandibular. Revista Gaúcha de Odontologia 1994;4(1):23-32.

7. Oliveira, AS, Dias EM, Contato RG, Berzin F. Prevalência de sinais e sintomas de disfunção temporomandibular em universitários brasileiros. Brazilian Oral Research 2006; 20(1):3-7.

8. Tosato JP, Caria PHF. Prevalência de DTM em várias faixas etárias. Revista Gaúcha de Odontologia 2006; 54(3):211-24.

9. Carlsson G, Le Reseche L. Epidemiology of temporomandibular disorders. In: Sessle B, Bryant $\mathrm{P}$, Dionne R. Progress in Pain Research and Management. IASP Press, 1995; p.211-26.

10. Vieira JC, Ferreira FM, Neto AJF, Simamoto Júnior PC, Teixeira M. Prevalência de disfunção temporomandibular entre universitários segundo índice de helkimo, e sua correlação com diversos hábitos parafuncionais; 2009. [Acesso em 2010 set 10]. Disponível em: http://www.icufu.org/cd2009/PDF/IC2009-0366.pdf

11. Fricton, JR et al. TMJ and craniofacial pain: diagnosis and management. St. Louis; 1988.

12. Ishigaki, S. et al. The distribution of internal derangement in patients with temporomandibular 
dysfunction: prevalence, diagnosis and

treatments. Cranio : The Journal of

Craniomandibular Practice 1992;10:289-96.

13. Scholte, AM et al. Characteristics and treatment outcome of diagnostic subgroups of CMD patients: retrospective study. Community Dentistry and Oral Epidemiology 1983;21:215-20. http://dx.doi.org/10.1111/j.1600-

\subsection{3.tb00759.x}

14. Pereira KNF, Andrade LLS, Costa MLG, Portal TF. Sinais e sintomas de pacientes com disfunção temporomandibular. Revista CEFAC 2005;7(2):221-8.

15. Pascoal MIN, Rapoport A, Chagas JFS, Pascoal MBN, Costa CC, Magna LA. Prevalência dos sintomas otológicos na desordem temporomandibular: estudo de 126 casos. Revista Brasileira Otorrinolaringologia 2001;67(5):627-33. http://dx.doi.org/10.1590/S0034-

\section{$\underline{72992001000500005}$}

16. Okeson JP. Dor orofacial: guia para avaliação, diagnóstico e tratamento. São Paulo; 1998.

17. Felício CM, Faria TG, Silva MAMR, Aquino AMCM, Junqueira CA. Desordem temporomandibular: relação entre sintomas otológicos e orofaciais. Revista Brasileira de Otorrinolaringologia 2004;70(6):786-93.

18. Melo AM. A interferência dos padrões mastigatórios nas disfunções temporomandibulares. Revista CEFAC 1998.

19. Palacios-Moreno AM, Chilvarquer I, Luz JGC. Achados radiográficos, sinais e sintomas nas disfunções da articulação temporomandibular. Revista de Odontologia da Universidade de São Paulo 1997;11(4):273-8.

20. Luz JGC, Oliveira NG. Incidence of temporomandibular joint disorders in patients seen at a hospital emergency room. Journal of Oral Rehabilitation 1994;21:349-51.

http://dx.doi.org/10.1111/j.1365-

2842.1994.tb01149.x

21. Bove SRK, Guimarães AS, Smith, RL. Caracterização dos pacientes de um ambulatório de disfunção temporomandibular e dor orofacial. Revista Latino-Americana de Enfermagem 2005; 13(5):686-91. 